\title{
Synchronization properties of network motifs: Influence of coupling delay and symmetry
}

Cite as: Chaos 18, 037116 (2008); https://doi.org/10.1063/1.2953582

Submitted: 21 February 2008 . Accepted: 09 June 2008 . Published Online: 22 September 2008

O. D’Huys, R. Vicente, T. Erneux, J. Danckaert, and I. Fischer

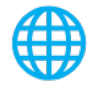

\section{ARTICLES YOU MAY BE INTERESTED IN}

Graph partitions and cluster synchronization in networks of oscillators

Chaos: An Interdisciplinary Journal of Nonlinear Science 26, 094821 (2016); https:// doi.org/10.1063/1.4961065

Low dimensional behavior of large systems of globally coupled oscillators

Chaos: An Interdisciplinary Journal of Nonlinear Science 18, 037113 (2008); https:// doi.org/10.1063/1.2930766

Cluster synchronization in oscillatory networks

Chaos: An Interdisciplinary Journal of Nonlinear Science 18, 037106 (2008); https:// doi.org/10.1063/1.2956986

\section{Don't let your writing} keep you from getting published! AIP $\mid$ Author Services

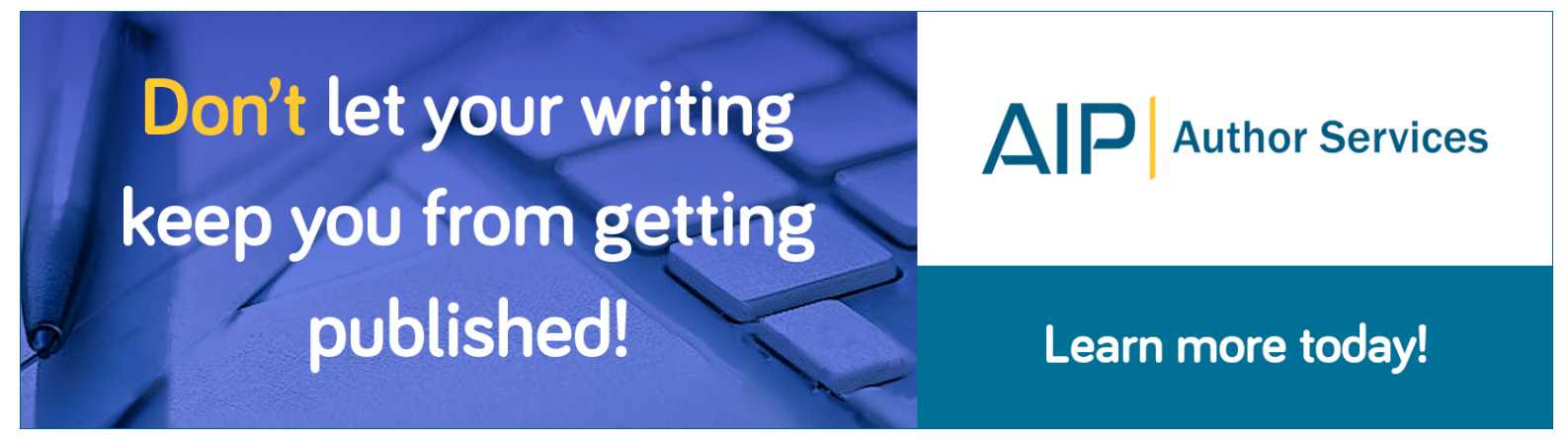




\title{
Synchronization properties of network motifs: Influence of coupling delay and symmetry
}

\author{
O. D’Huys, ${ }^{1}$ R. Vicente, ${ }^{2,3}$ T. Erneux, ${ }^{4}$ J. Danckaert, ${ }^{1}$ and I. Fischer ${ }^{5}$ \\ ${ }^{1}$ Department of Physics (DNTK) and Department of Applied Physics and Photonics (TONA), \\ Vrije Universiteit Brussel, Pleinlaan 2, 1050 Brussels, Belgium \\ ${ }^{2}$ Max-Planck-Institute for Brain Research, Deutschordenstraße 46, 60528 Frankfurt am Main, Germany \\ ${ }^{3}$ Frankfurt Institute for Advanced Studies (FIAS), Ruth-Moufang-Straße 1, \\ 60438 Frankfurt, Germany \\ ${ }^{4}$ Université Libre de Bruxelles, Optique Nonlinéaire Théorique, Campus Plaine, C.P. 231, \\ Boulevard du Triomphe, 1050 Bruxelles, Belgium \\ ${ }^{5}$ School of Engineering and Physical Sciences, Heriot-Watt University, Edinburgh EH1 4AS, \\ Scotland, United Kingdom
}

(Received 21 February 2008; accepted 9 June 2008; published online 22 September 2008)

\begin{abstract}
We investigate the effect of coupling delays on the synchronization properties of several network motifs. In particular, we analyze the synchronization patterns of unidirectionally coupled rings, bidirectionally coupled rings, and open chains of Kuramoto oscillators. Our approach includes an analytical and semianalytical study of the existence and stability of different in-phase and out-ofphase periodic solutions, complemented by numerical simulations. The delay is found to act differently on networks possessing different symmetries. While for the unidirectionally coupled ring the coupling delay is mainly observed to induce multistability, its effect on bidirectionally coupled rings is to enhance the most symmetric solution. We also study the influence of feedback and conclude that it also promotes the in-phase solution of the coupled oscillators. We finally discuss the relation between our theoretical results on delay-coupled Kuramoto oscillators and the synchronization properties of networks consisting of real-world delay-coupled oscillators, such as semiconductor laser arrays and neuronal circuits. () 2008 American Institute of Physics.
\end{abstract}

[DOI: $10.1063 / 1.2953582$ ]

Complex networks are studied in scientific fields varying from social sciences to biology. Network motifs are major constituents of larger networks of oscillators and the determination of their properties can provide an intermediate step to better understand the emergent behaviors in larger collections. One of those dynamical processes is the synchronization phenomenon. A coupling delay may naturally appear in the interaction between the nodes, as the propagation time of a signal. This delay may modify dynamics drastically, giving rise to new collective behavior and different synchronization patterns. In this paper we study the synchronization patterns, concentrating on periodic solutions appearing in unidirectional rings, bidirectional rings and open chains of Kuramoto oscillators; patterns of interconnectedness which appear as network motifs. We show that some networks show a tendency to adjust their phases in the presence of coupling delays, while in other configurations nonuniform states are as likely as the fully synchronized state. We then try to relate our results on Kuramoto oscillators to the synchronization behavior observed networks of semiconductor lasers and neuronal circuits.

\section{INTRODUCTION}

Complex systems of diverse origin are often organized in topologies with global statistical properties that are qualitatively different from those of random or perfectly ordered structures. ${ }^{1,2}$ The study of these architectures of interactions involving many nodes has gained considerable interest in fields ranging from social sciences to systems biology. ${ }^{3,4} \mathrm{~A}$ multitude of complex networks define relations between nodes which are not static entities but may even follow their own dynamical rules of evolution. The topology of the network turns out to be a fundamental ingredient in defining the collective properties of the system dynamics. ${ }^{5,6}$

The synchronization phenomenon is one of the most important examples of a self-organized dynamical process occurring in such coupled systems. ${ }^{7,8}$ Synchronization, being in general the adjustment of rhythms between interacting elements, has been experimentally or numerically reported in brain activity, ${ }^{9,10}$ laser arrays, ${ }^{11,12}$ Josephson junctions, ${ }^{13,14}$ coupled logistic maps ${ }^{6,15-17}$ or social behavior, ${ }^{18}$ to mention a few examples. In many cases the appearance of synchronization is considered to be a fundamental property of the system. ${ }^{19}$ Furthermore, synchronization of complex dynamics has proven to be attractive and essential for practical applications. ${ }^{20}$

A major contribution to understand the design principles and dynamics of complex networks was the proposal to decompose such structures into the small repeating patterns of interconnectedness that conform the network, the so-called network motifs. ${ }^{21}$ The analysis of which and how often these building blocks (typically made of only few nodes) appear in a given network allows us to gain insight into the network function and rules of evolution. ${ }^{22,23}$ Moreover, network mo- 
tifs may constitute a basis to define universal classes of networks in spite of their very different origin.

In this article we analyze the fundamental properties of synchronization of several of these networks motifs. Global dynamics can in general be affected by the symmetry of the topological arrangement of such elements. ${ }^{5,24-27}$ In particular, we investigate the synchronization solutions of delaycoupled oscillators arranged in unidirectional and bidirectional rings as well as those of open chains. These modules are major constituents of larger networks of oscillators and the determination of their properties can provide an intermediate step to better understand the emergent behaviors in larger collections of coupled oscillators. ${ }^{28}$ Temporal latencies arising as a consequence of the finite propagation velocity of interactions (for example, when a given perturbation needs to propagate along the physical separation between systems) sometimes have to be explicitly taken into account in the coupling between oscillators. This is always the case, when the coupling times become comparable or even larger than the oscillation time scales of the individual oscillators, for example, for coupled semiconductor lasers or connected neuronal assemblies in the brain. Mathematically, introducing a delay into the coupling between differential equations transforms the finite system into an infinite-dimensional one. We focus here on the influence of coupling delays and symmetry on the synchronization patterns arising in networks of oscillators. ${ }^{29}$

The paper is organized as follows: In the next section we introduce the modeling equations of Kuramoto oscillators and present the basic notation. In Sec. III we reconsider the case of two mutually delay-coupled Kuramoto oscillators and extend the analysis to open chains and arrays with delayed feedback. We continue with an analysis of unidirectionally delay-coupled rings of phase oscillators in Sec. IV. There, we establish a general stability criterion for both inphase and out of phase types of frequency-locked solutions. Bidirectional rings are investigated in Sec. V, where we describe how the coupling delay can act as a symmetryrestoring parameter. Finally, we discuss our results on the context of delay-coupled systems and specific applications in laser dynamics and neuroscience.

\section{NETWORKS OF KURAMOTO OSCILLATORS WITH TIME DELAY}

In our analysis, a Kuramoto system is used to describe the oscillatory dynamics of each node in the network. We have chosen this approach for two main reasons. First, phase-reduction techniques show that for weak coupling strengths several important classes of interacting oscillators can be approximated by Kuramoto equations. ${ }^{30,31}$ Second, such simple and generic model of limit-cycle oscillators allowed us to perform some analytical calculations. In the next sections we describe how the symmetry of the network modules and the delay determine the basic synchronization solutions into which the oscillators can self-organize.

Our networks consist of identical Kuramoto phase oscillators which topology is represented by the adjacency matrix $A$ and which dynamics follows the equation

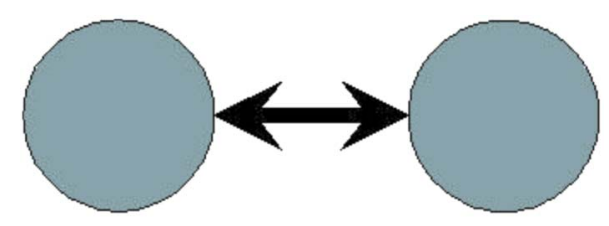

FIG. 1. (Color online) A schematic representation of two mutually coupled oscillators.

$$
\theta_{i}^{\prime}(t)=\omega_{0}+\frac{K}{q} \sum_{j} A_{i j} \sin \left[\theta_{j}(t-T)-\theta_{i}(t)\right],
$$

where $\theta_{i}$ represents the phase variable, $K$ stands for the coupling strength, $q$ is the degree of the node $i$, the prime denotes derivation with respect to time, and $T$ is the transmitting delay associated with the noninstantaneous interaction between oscillators.

Schuster and Wagner were the first to study two mutually coupled Kuramoto elements in the presence of a coupling delay. ${ }^{24}$ The delay was then found to act as an inducer of multiple frequency-locked solutions. Yeung and Strogatz extended this study to another elemental configuration. They investigated a population of globally coupled oscillators interacting with time delay and established stability criterion for the fully synchronous solutions as well as locked and disordered states. ${ }^{32}$ In the context of delay-coupled semiconductor lasers, Kozyreff, Vladimirov, and Mandel studied globally coupled networks of a finite number of Kuramoto oscillators. ${ }^{33}$

Another major result is reported by Earl and Strogatz: ${ }^{34}$ a stability criterion is derived for a uniform state, valid for any delay-coupled network of identical phase oscillators with a constant degree and an arbitrary coupling function $f$. The synchronous state, with locking frequency $\Omega$, in the presence of a coupling delay $T$ is stable if and only if

$$
K f^{\prime}(-\Omega T)>0,
$$

where $f^{\prime}$ denotes the derivative of $f$ with respect to time. For sinusoidally coupled oscillators, as in the Kuramoto case, this criterion reads

$$
K \cos (\Omega T)>0 .
$$

The stability of an in-phase periodic solution hence only depends on the coupling function and the delay and not on the details of the network topology. However, the ability of a network to show total synchronization does not only depend on the occurrence and stability of this uniform solution, but also on the existence and stability of competing nonuniform solutions. Such states are determined by the network topology and the delay. These parameters can thus modify the collective behavior of the network as we shall see in the following.

\section{TWO MUTUALLY COUPLED KURAMOTO OSCILLATORS}

We first reconsider the system studied by Schuster and Wagner: ${ }^{24}$ two Kuramoto oscillators coupled with delay. A sketch of this configuration is shown in Fig. 1. Schuster and Wagner considered two detuned oscillators, ${ }^{24}$ while we as- 
sume the elements to be identical, as we are interested in networks possessing a symmetry. The dynamical behavior is modelled by

$$
\begin{aligned}
& \theta_{1}^{\prime}(t)=\omega_{0}+K \sin \left[\theta_{2}(t-T)-\theta_{1}(t)\right], \\
& \theta_{2}^{\prime}(t)=\omega_{0}+K \sin \left[\theta_{1}(t-T)-\theta_{2}(t)\right] .
\end{aligned}
$$

We can rescale the variables, eliminating the natural frequency $\omega_{0}$,

$$
\kappa=\frac{K}{\omega_{0}}, \quad \tau=\omega_{0} T .
$$

The equation of motion (4) becomes

$$
\begin{aligned}
& \dot{\theta}_{1}(t)=1+\kappa \sin \left[\theta_{2}(t-\tau)-\theta_{1}(t)\right], \\
& \dot{\theta}_{2}(t)=1+\kappa \sin \left[\theta_{1}(t-\tau)-\theta_{2}(t)\right],
\end{aligned}
$$

where time is measured in units of $\omega_{0} t$.

Without delay Eq. (5) can be reduced to the Adler equation. If the coupling strength $\kappa$ is chosen positive, the coupling is attractive. The oscillators will evolve to a symmetric state $\theta_{1}(t)=\theta_{2}(t)=t$. If $\kappa<0$, i.e., the oscillators are coupled repulsively, they tend towards an antiphase oscillation $\theta_{1}(t)=\theta_{2}(t)+\pi=t$.

In the presence of a coupling delay, the system still exhibits frequency-locked symmetric and antisymmetric states. However, due to the delayed coupling, multiple solutions with different locking frequencies become possible. For the in-phase state, these frequencies can be found by solving

$$
\omega=1-\kappa \sin (\omega \tau) .
$$

The stability of these solutions is given by Eq. (3), with $\Omega=\omega_{0} \omega$. The locking frequencies corresponding to the antisymmetric solutions, are implicitly given by

$$
\omega=1+\kappa \sin (\omega \tau) .
$$

The stability of the antiphase solutions can be determined analogously to the method used by Earl and Strogatz. ${ }^{34}$ An antiphase solution is stable if and only if

$$
\kappa \cos \omega \tau<0 \text {. }
$$

Stable in-phase and antiphase solutions are thus exchanged when the sign of the coupling strength $\kappa$ is reversed, but this is only due to the symmetry of the coupling function. In Fig. 2 the different locking frequencies are shown as a function of the delay time. Branches of stable symmetric and antisymmetric frequencies alternate with each other, both varying from $1+|\kappa|$ to $1-|\kappa|$. In Fig. 3 the stable parameter ranges are plotted for both states. As long as $\tau<\pi /[2(1$ $+|\kappa|)]$, the delay does not significantly alter the dynamical behavior. For small coupling strength, the system will jump from one solution to the other for increasing coupling delay, while for stronger coupling multiple solutions coexist. The delay here mainly induces multistability.
(1)

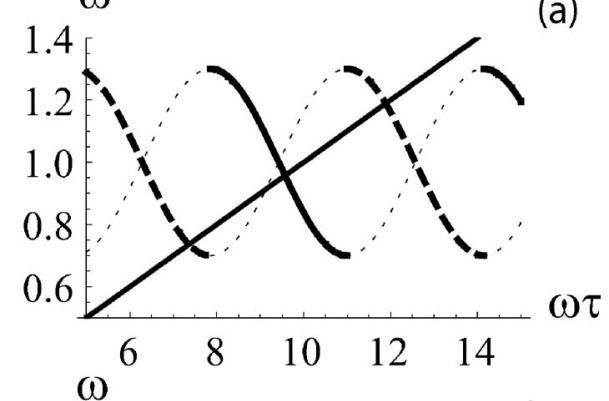

(b)

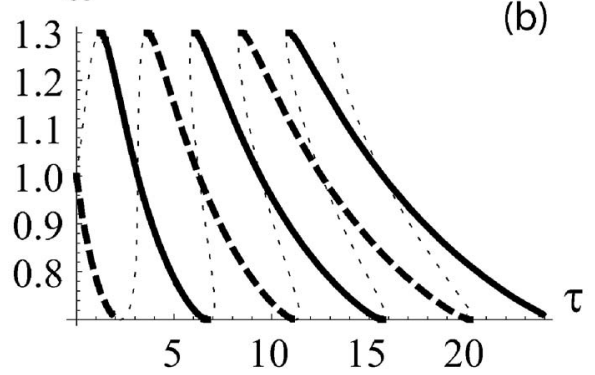

FIG. 2. (a) Shows a graphical determination of the locking frequencies, given by Eqs. (6) and (7), for two mutually coupled elements. The intersections with the full black curve correspond to stable in-phase solutions, those with the dashed curves to stable antiphase states. Intersections with the dotted lines correspond to unstable locking frequencies. The parameters were chosen as $\kappa=-0.3$ and $\tau=10$. In (b) the locking frequencies of two mutually coupled Kuramoto oscillators are shown for varying delay, and a coupling strength of $\kappa=-0.3$. The full curve corresponds to stable in-phase solutions, the dashed curves to stable antiphase solutions, and the dotted lines represent unstable frequency-locked solutions.

\section{A. Open chains of Kuramoto oscillators}

We have also studied a generalization of the former configuration by analyzing an arbitrarily number of elements interacting bidirectionally in an open chain topology. $n$ Kuramoto oscillators arranged in such geometry can be described by

$\dot{\theta}_{1}(t)=1+\kappa \sin \left[\theta_{2}(t-\tau)-\theta_{1}(t)\right]$

$\dot{\theta}_{i}(t)=1+\frac{\kappa}{2}\left\{\sin \left[\theta_{i+1}(t-\tau)-\theta_{i}\right]+\sin \left[\theta_{i-1}(t-\tau)-\theta_{i}(t)\right]\right\}$,

$\dot{\theta}_{n}(t)=1+\kappa \sin \left[\theta_{n-1}(t-\tau)-\theta_{n}(t)\right]$,

where $2 \leqslant i \leqslant n-1$. The inner oscillators are coupled with only half of the individual coupling strength, as they are

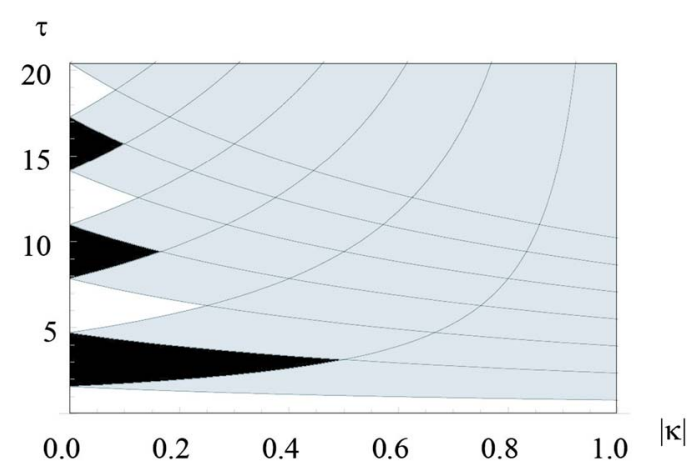

FIG. 3. (Color online) Stable regions in parameter space, for two mutually Kuramoto oscillators. Stability is calculated using Eqs. (3) and (8). The coupling is repulsive. Black: only stable in-phase solutions exist; white: only stable antiphase solutions exist; gray: the two states coexist. 


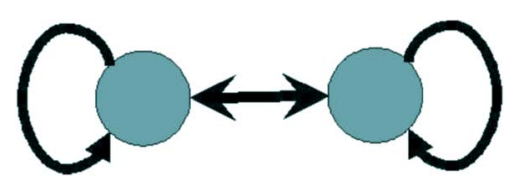

FIG. 4. (Color online) Sketch of two oscillators coupled with feedback.

connected to two other elements. The mathematical analysis is similar to those of only two coupled elements: all oscillators move periodically either in-phase with each other, or the even-numbered oscillators are antiphase with the oddnumbered ones. The stable locking frequencies for both states are the same as for just two coupled elements. If we do not scale the coupling strength of the inner oscillators, the outer ones show a small phase offset (upon the possible phase difference of $\pi$ ) with respect to the middle oscillators. This phase difference originates from the fact that the outer elements receive less input than the inner ones.

In particular, in a chain of three elements, in the in-phase solution all three oscillators are in phase, while in the antiphase state the two outer oscillators are in antiphase with the middle one (and in-phase with each other). Consequently, the synchronization between two elements coupled through a third relay element - or equivalently, between the outer elements in a chain of three-is stronger than between two (directly) coupled elements. In-phase and antiphase solutions can coexist in the latter configuration, while in a chain of three elements the outer elements will always synchronize in-phase, regardless of whether the coupling is repulsive or attractive, for any value of the coupling strength and the coupling delay. Our numerical simulations indicate that even a detuning of the middle oscillator with respect to the outer ones does not destroy the symmetric behavior.

\section{B. The influence of feedback}

As a next step we add a delayed feedback term to Eqs. (5). In Fig. 4 the scheme of two oscillators interacting in this topology is represented. We consider the same values for the coupling delay and the feedback delay.

The system is then modelled by

$$
\begin{aligned}
\dot{\theta}_{1}(t)= & 1+\frac{\kappa}{2}\left\{\sin \left[\theta_{2}(t-\tau)-\theta_{1}(t)\right]\right. \\
& \left.+\sin \left[\theta_{1}(t-\tau)-\theta_{1}(t)\right]\right\}, \\
\dot{\theta}_{2}(t)= & 1+\frac{\kappa}{2}\left\{\sin \left[\theta_{1}(t-\tau)-\theta_{2}(t)\right]\right. \\
& \left.+\sin \left[\theta_{2}(t-\tau)-\theta_{2}(t)\right]\right\} .
\end{aligned}
$$

With feedback the system has a stronger symmetry than without feedback; the oscillators always get the same input, no matter if they are in phase or not, while in Eq. (5) the components only get the same input provided that they are in-phase or antiphase.

The system with feedback only admits synchronous or antisynchronous frequency-locked solutions, just as the system without feedback. For the in-phase solutions, the locking frequencies do not change due to the introduction of the

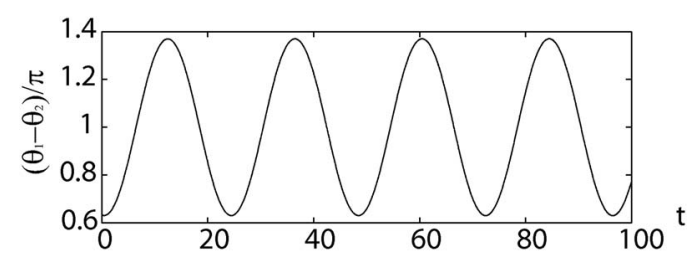

FIG. 5. The time trace of $\left[\theta_{1}(t)-\theta_{2}(t)\right] / \pi$ is plotted, for two delay-coupled Kuramoto oscillators with feedback [as described by Eq. (10)]. The coupling delay is chosen as $\tau=6$ and the coupling strength is chosen as $\kappa=-0.3$. The period of the oscillations is 24 .

feedback; a linear stability analysis leads to the same stability criterion, Eq. (3). But when the oscillators are in antiphase, the two coupling terms cancel in Eq. (10), resulting in only one locking frequency $\omega=1$.

A linear stability analysis around the antiphase solution leads to the following characteristic equation:

$$
\begin{gathered}
\left(2 \lambda-\kappa \cos \tau e^{-\lambda \tau}\right)^{2}=\kappa^{2} \cos ^{2} \tau e^{-2 \lambda \tau} \\
\Leftrightarrow \lambda=\kappa \cos \tau e^{-\lambda \tau} \\
\text { or } \quad \lambda=0 .
\end{gathered}
$$

The zero-root corresponds to global phase changes. For zero delay the antisynchronous state is stable if $\kappa<0$. For nonzero delay and nonzero root, we separate Eq. (11) in a real and imaginary part

$$
\begin{gathered}
\operatorname{Re}(\lambda) \equiv \mu=\kappa \cos \tau e^{-\mu \tau} \cos (\sigma \tau), \\
\operatorname{Im}(\lambda) \equiv \sigma=-\kappa \cos \tau e^{-\mu \tau} \sin (\sigma \tau) .
\end{gathered}
$$

If $\kappa \cos \tau>0$, we can always choose $\sigma=0$. In this case Eq. (13) always has a positive solution, meaning the antiphase solution is unstable. If $\kappa \cos \tau<0$, stability depends on the factor $\cos \sigma \tau$. From Eq. (14) it is clear that a solution for which $\cos \sigma \tau<0$ is only possible if $-\kappa \tau \cos \tau \geqslant \pi / 2$. The antiphase state hence loses and regains stability in a Hopf bifurcation if $\cos (\sigma \tau)=0$ and $\sigma \tau=\pi / 2=-\kappa \tau \cos \tau$. Numerically we find for some parameter sets oscillatory solutions emerging at this Hopf bifurcation point. (Numerical simulations were performed with "Dynamics Solver" by J. M. Aguirregabiria. We used a Runge-Kutta 4 integration code, with a step size of 0.001.) The two oscillators have the same mean frequency, but the phase difference varies periodically with a period of $4 \tau$, as shown in Fig. 5. The origin of this solution can be understood analytically: the limit cycle oscillation period at the Hopf bifurcation point is given by $2 \pi / \sigma=4 \tau$.

In the presence of a mismatch between feedback delay time and coupling delay time, and between feedback strength and coupling strength, exact in-phase and antiphase periodic solutions are still found numerically. Similar oscillations are also observed if the mismatches are small. The oscillation period is then not any more exactly equal to four times the coupling delay. However, the locked antiphase solution gains stability as the mismatch grows.

The parameter ranges which admit stable in-phase or antiphase frequency-locked solutions, are plotted in Fig. 6. For small values of the coupling strength, the two states still alternate with respect to each other when we increase the 


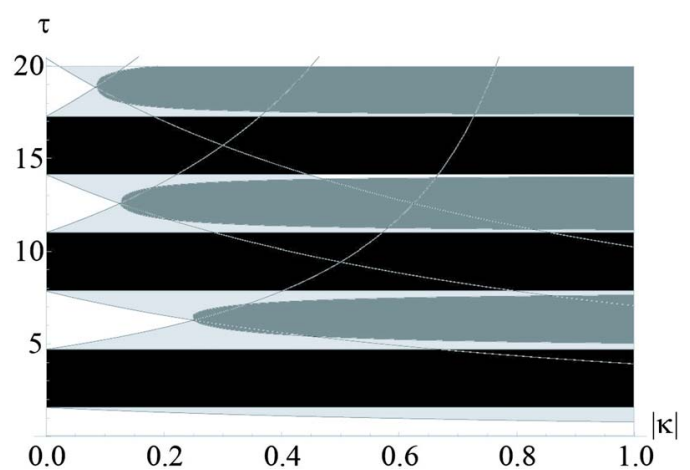

FIG. 6. (Color online) Parameter sets allowing stable in-phase or antiphase frequency-locked solutions, and oscillatory solutions, for two delay-coupled Kuramoto oscillators with feedback. The coupling is chosen to be repulsive. Stability is calculated using Eq. (3) for the in-phase state and Eq. (11) for the antiphase state. Black: only in-phase solutions are stable; white: only antiphase solutions are stable; gray: the two types of solutions coexist; dark gray: stable in-phase solutions may coexist with oscillatory solutions.

delay, just like in the situation without feedback. However, the in-phase states are stable for a larger range of delay times than the antiphase states. Between the Hopf bifurcation lines antiphase frequency-locked solutions are unstable, but stable oscillatory (unlocked) solutions may coexist with in-phase locked solutions.

For small delays the solutions of the two networks consisting of two components are similar with and without feedback. However, antiphase frequency-locked solutions are only stable for small delays, or small coupling strength. For larger coupling strength and delay we may find oscillatory solutions, which always coexist with in-phase solutions. In this network the delay can be seen as a symmetrizing parameter, as the in-phase solutions are stable for considerable larger areas in parameter space than the antiphase and oscillatory solutions.

\section{UNIDIRECTIONAL RINGS}

We continue with a unidirectional ring of $n$ Kuramoto oscillators, where each oscillator is coupled to exactly one of its neighbors. A schematic representation of such networks is shown in Fig. 7. This network is invariant under rotations by $2 k \pi / n$; its symmetry group is the cyclic group $\mathbb{Z}_{n}$. The system is described by the following equation:

$$
\dot{\theta}_{i}=1+\kappa \sin \left[\theta_{i+1}(t-\tau)-\theta_{i}(t)\right]
$$

where $n+1 \equiv 1$.

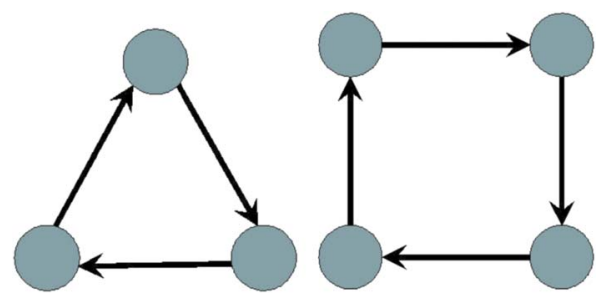

FIG. 7. (Color online) Unidirectionally coupled rings of three and four elements.
This network admits $n$ different frequency-locked solutions

$$
\theta_{k}(t)=\omega t+k \Delta \phi \quad \text { with } \Delta \phi=\frac{2 j \pi}{n}, \quad 0 \leqslant j<n .
$$

If $j=0$, all oscillators move in-phase, and the dynamics exhibit the full network symmetry. The other solutions are characterized by spatiotemporal symmetries, as the system is invariant under the combination of a rotation and a time shift. Although other, unlocked, solutions cannot be excluded, these were the only ones we found numerically.

The locking frequency $\omega$ is then implicitly given by

$$
\omega=1+\kappa \sin (\Delta \phi-\omega \tau) \text {. }
$$

In order to obtain information about the stability of the different solutions, we perform a linear stability analysis. The evolution of a small perturbation $\delta(t) \propto e^{\lambda t}$ to the solution (17) is described by

$$
\dot{\delta}_{l}(t)=\kappa \cos (\Delta \phi-\omega \tau)\left[-\delta_{l}(t)+e^{-\lambda \tau} A_{l m} \delta_{m}(t)\right],
$$

where $A$ denotes the adjacency matrix

$$
A=\left(\begin{array}{llll}
0 & 1 & 0 & \cdots \\
0 & 0 & 1 & \cdots \\
\vdots & & & \ddots \\
1 & 0 & 0 & \cdots
\end{array}\right) .
$$

We will not consider the case $\cos (\Delta \phi-\omega \tau)=0$. In this limit case a nonlinear stability analysis is required.

The adjacency matrix $A$ has eigenvectors $(1, \ldots, 1)$, with eigenvalue 1 , and $[1, \exp (2 m \pi i / n), \exp (4 m \pi i / n), \ldots]$ with eigenvalues $\exp (2 m \pi i / n)$. The first eigenmode corresponds to global phase changes and is neutrally stable for any value of the coupling delay. The other eigenmodes represent traveling waves along the ring. If we now apply a perturbation along such an eigenmode, we obtain for the eigenvalues of the characteristic equation (18),

$$
\lambda=\kappa \cos (\Delta \phi-\omega \tau)\left(-1+e^{-\lambda \tau} e^{2 m \pi i / n}\right) .
$$

For zero delay, a solution is stable if $\kappa \cos \Delta \phi>0$. The growth rate $\lambda$ depends continuously on the delay time $\tau$. If a state loses stability, the values of $\omega$ and $\tau$ must correspond to a pure imaginary eigenvalue $\lambda=i \sigma$. As the characteristic equation (20) does not admit such solutions, we can establish a stability criterion: A state $\theta_{k}(t)=\omega t+k \Delta \phi$ is stable if and only if

$$
\kappa \cos (\Delta \phi-\omega \tau)>0 .
$$

If $\Delta \phi=0$, this criterion reduces to the criterion for the inphase solution, Eq. (3). For $\Delta \phi=\pi$, we find the criterion for the antisymmetric state in a network of two coupled Kuramoto oscillators, Eq. (8).

This result can easily be generalized; if the phase oscillators are coupled through an arbitrary function $f$, an (antiphase) state is stable if and only if $\kappa f^{\prime}(\Delta \phi-\omega \tau)>0$. We can also prove Eq. (21) following the method of Earl and Strogatz. ${ }^{34}$

The different states obey a very similar stability criterion, the different solutions are stable over comparable pa- 
$\omega$

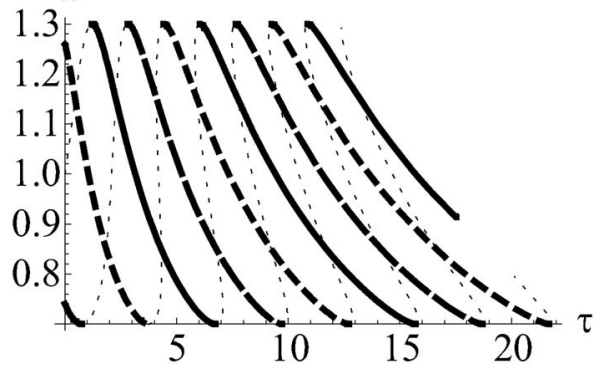

FIG. 8. The locking frequencies [determined by Eq. (17)] for a unidirectionally coupled ring of three Kuramoto oscillators are plotted for varying delay, and a coupling strength $\kappa=-0.3$. The full curve corresponds to stable in-phase solutions, the dashed curve to stable out-of-phase solutions with $\Delta \phi=4 \pi / 3$, the curve with the long dashes represents stable out-of-phase solutions with $\Delta \phi=2 \pi / 3$, and the dotted lines correspond to unstable frequency-locked solutions. The stability of the locking frequencies is derived from Eq. (21).

rameter ranges. The delay mainly acts as an inducer of multiple solutions; it does not enhance the stability of one of the different states. Consequently, uniform synchronization is not more likely than the less symmetric synchronization states. To illustrate this we examine the behavior of unidirectional rings of three and four elements. Figure 8 represents the different locking frequencies for a ring of three elements for a fixed coupling strength. For each of the three different states $(\Delta \phi=0, \Delta \phi=2 \pi / 3$, and $\Delta \phi=4 \pi / 3)$, the frequency varies continuously from $1+|\kappa|$ to $1-|\kappa|$ for increasing delay, without changing its stability. Each state has on average the same number of stable locking frequencies. In the bifurcation diagram of a unidirectional ring of four elements, as shown in Fig. 9, the four different states show the same characteristics.

Figure 10 shows the sets of parameters allowing stable frequency-locked solutions. For small values of the coupling strength or the delay only one or two solutions coexist, while for larger values the different states coexist. In comparison to a ring of only two elements (as shown in Fig. 3), introducing more elements leads to a richer spectrum, with more coexisting states.

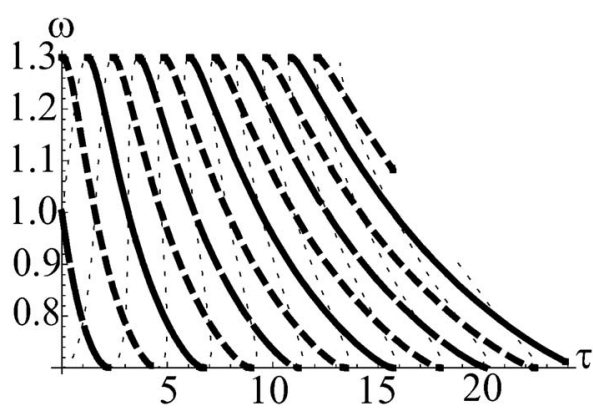

FIG. 9. The different locking frequencies are shown for varying delay, for a ring of four unidirectionally coupled Kuramoto oscillators. The coupling strength is chosen to be $\kappa=-0.3$. The full curve corresponds to stable inphase solutions, the dashed curves to stable out-of-phase solutions with $\Delta \phi= \pm \pi / 2$ and the long dashed curve represents the antisymmetric state $\Delta \phi=\pi$. The dotted lines represent unstable frequency-locked solutions. The locking frequencies are calculated using Eq. (17), and their stability is given by Eq. (21).

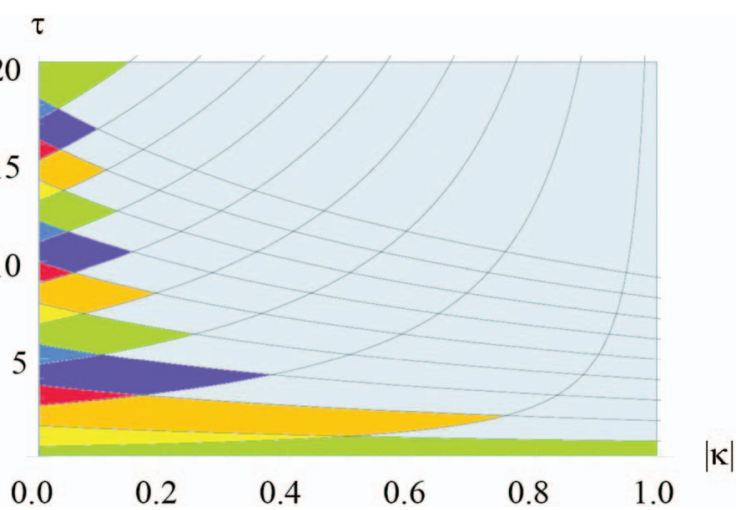

FIG. 10. (Color) Stable regions in parameter space, for a ring of three unidirectionally coupled Kuramoto oscillators. Stability is calculated using Eqs. (17) and (21). The coupling is repulsive. Red: only stable in-phase solutions exist; blue: only stable out-of-phase solution exist $\Delta \phi=2 \pi / 3$; yellow: only stable out-of-phase solutions exist, $\Delta \phi=4 \pi / 3$; green: both types of out-of-phase solutions coexist; orange: the solutions $\Delta \phi=2 \pi / 3$ and $\Delta \phi$ $=0$ coexist; purple: the solutions $\Delta \phi=4 \pi / 3$ and $\Delta \phi=0$ coexist; gray: the three solutions coexist.

\section{BIDIRECTIONAL RINGS}

In a bidirectional ring each oscillator is coupled to its two neighbors. The network has both rotational and mirror symmetry, described by the dihedral group $D_{n}$. In Fig. 11 a sketch of such networks is shown. The dynamical behavior is modelled by

$$
\begin{aligned}
\dot{\theta}_{i}(t)= & 1+\frac{\kappa}{2}\left\{\sin \left[\theta_{i+1}(t-\tau)-\theta_{i}(t)\right]\right. \\
& \left.+\sin \left[\theta_{i-1}(t-\tau)-\theta_{i}(t)\right]\right\},
\end{aligned}
$$

where $1 \equiv n+1$.

We investigate the same frequency-locked solutions as in the unidirectional ring

$$
\theta_{k}(t)=\omega t+k \Delta \phi \quad \text { with } \quad \Delta \phi=\frac{2 j \pi}{n}, \quad j \leqslant \frac{n}{2} .
$$

Due to the mirror symmetry of the network, a solution is invariant when we change the sign of $\Delta \phi$. We can hence only distinguish half as much different out-of-phase states as for the unidirectional ring.

We find the locking frequency $\omega$ by solving the equation,

$$
\omega=1-\kappa \sin (\omega \tau) \cos \Delta \phi .
$$
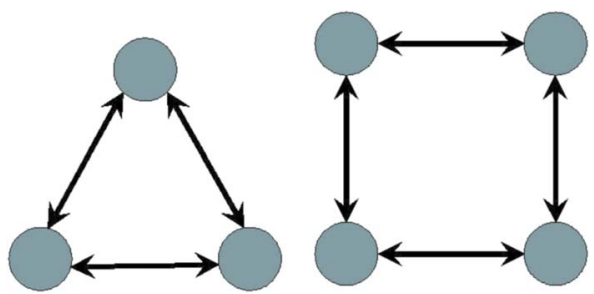

FIG. 11. (Color online) Sketch of three and four bidirectionally coupled oscillators. 
While in unidirectional rings we find on average the same number of locking frequencies for each state for a fixed coupling delay, the number of locking frequencies of a state scales with $\cos \Delta \phi$ in a bidirectional ring.

We apply a linear stability analysis and obtain the following dynamics for a small perturbation $\delta(t)$ to the solution (23):

$$
\dot{\delta}_{l}(t)=\kappa \cos \Delta \phi \cos \omega \tau\left[-\delta_{l}(t)+e^{-\lambda \tau} B_{l m} \delta_{m}(t)\right],
$$

with

$$
B=\frac{1}{2}\left(\begin{array}{ccccc}
0 & 1+x & \cdots & 0 & 1-x \\
1-x & 0 & 1+x & \cdots & 0 \\
& \ddots & \ddots & \ddots & \\
1+x & 0 & \cdots & 1-x & 0
\end{array}\right)
$$

and $x=\tan \Delta \phi \tan \omega \tau$.

The matrix $B$ does not coincide with the adjacency matrix of the network in this case. $B$ has eigenvectors $\{1, \exp (2 i k \pi / n), \ldots, \exp [2 i(n-k) \pi / n]\}$ with eigenvalues $\cos (2 k \pi / n)+i x \sin (2 k \pi / n)$ and the eigenvector $(1, \ldots, 1)$ with eigenvalue 1 (corresponding to global phase changes).

Again, we perform a stability analysis along an eigenvector and obtain the following equation for the eigenvalue $\lambda$,

$$
\begin{aligned}
\lambda= & \kappa \cos \Delta \phi \cos \omega \tau\left[-1+e^{-\lambda \tau}\left(\cos \frac{2 k \pi}{n}\right.\right. \\
& \left.\left.+i \tan \omega \tau \tan \Delta \phi \sin \frac{2 k \pi}{n}\right)\right] .
\end{aligned}
$$

Without delay, a solution is stable if $\kappa \cos \Delta \phi>0$. However, a solution may lose stability through a Hopf bifurcation as we increase the delay continuously. We can already note that a Hopf bifurcation is not possible if $|\tan \omega \tau \tan \Delta \phi|<1$.

We next investigate two particular values of $\Delta \phi$. A solution $\Delta \phi=\pi$ refers to a state where each oscillator moves in phase with its second nearest neighbors and in antiphase with its nearest neighbors. It occurs if the ring has an even number of oscillators. The characteristic equation (26) becomes

$$
\lambda=\kappa \cos \omega \tau\left(1-e^{-\lambda \tau} \cos \frac{2 k \pi}{n}\right) .
$$

A pure imaginary solution is impossible; the eigenvalues do not cross the imaginary axis. This solution is hence stable if and only if $\kappa \cos \omega \tau<0$.

Configurations with $\Delta \phi= \pm \pi / 2$ occur in rings where the number of oscillators $n$ is a multiple of four. Stability is determined by the equation

$$
\lambda=i \kappa e^{-\lambda \tau} \sin \omega \tau \sin \frac{2 k \pi}{n} .
$$

We can choose $2 k \pi / n=\pi / 2$. Decoupling Eq. (28) in a real and imaginary part $\lambda=\mu+i \sigma$ yields

$$
\begin{aligned}
& \mu=\kappa \sin \omega \tau e^{-\mu \tau} \sin \sigma \tau, \\
& \sigma=\kappa \sin \omega \tau e^{-\mu \tau} \cos \sigma \tau .
\end{aligned}
$$

If $\kappa \sin \omega \tau$ is positive, Eq. (30) has a solution for which $0<\sigma \tau<\pi / 2$. If $\kappa \sin \omega \tau$ is negative, Eq. (30) has a solution for which $-\pi / 2<\sigma \tau<0$. There is hence always a root for which $\kappa \sin \omega \tau \sin \sigma \tau>0$, which means Eq. (29) has at least one positive solution. A state with $\Delta \phi= \pm \pi / 2$ is thus always unstable for nonzero delay.

For other values of $\Delta \phi$ the solution may lose stability through a Hopf bifurcation as we increase the delay. For small coupling strengths a Hopf bifurcation occurs for

$$
\tau=\left(\frac{\pi}{2}+2 m \pi\right)\left[1+\kappa \frac{\cos ^{2} \Delta \phi-\left(1+\cos \frac{2 \pi}{n}\right) \sin ^{2} \Delta \phi}{\cos \Delta \phi}\right]
$$

and for

$$
\begin{aligned}
\tau= & \left(\frac{3 \pi}{2}+2 m \pi\right) \\
& \times\left[1-\kappa \frac{\cos ^{2} \Delta \phi-\left(1+\cos \frac{2 \pi}{n}\right) \sin ^{2} \Delta \phi}{\cos \Delta \phi}\right] .
\end{aligned}
$$
if

When the product $\kappa \tau$ is large, we find a Hopf bifurcation

$\tan \omega \tau \tan \Delta \phi= \pm 1$

All together, we obtain a sufficient stability criterion for the antiphase states: if

$$
\kappa \cos (\omega \tau+\Delta \phi)>0 \quad \text { and } \kappa \cos (\omega \tau-\Delta \phi)>0,
$$

a state $\theta_{k}(t)=\omega t+k \Delta \phi$ is stable. The criterion is also valid as a necessary condition in the limit of long delays and strong couplings.

In contrast to the unidirectional ring, the delay has a different effect on the stability of the in-phase and out-ofphase states. If the number of elements in the ring is even, we find two preferred states: the synchronous $(\Delta \phi=0)$ and the antisynchronous $(\Delta \phi=\pi)$. If the ring has an odd number of elements, the uniform solution will be the most likely state.

As an example, we consider a ring of three bidirectionally coupled Kuramoto oscillators. In Fig. 12 the different locking frequencies are depicted as a function of the coupling delay. The most symmetric solution dominates as the branches of locking frequencies evolve over a larger range. Moreover, while the in-phase solution is stable over the whole branch, the out-of-phase solution undergoes a Hopf bifurcation. The stability of the out-of-phase state is determined using Eq. (26). Figure 13 shows the sets of parameters allowing stable in-phase and out-of-phase states. The Hopf bifurcation point is well approximated by its large delay limit equation (33). Further, the in-phase state is stable for a larger 
$\omega$

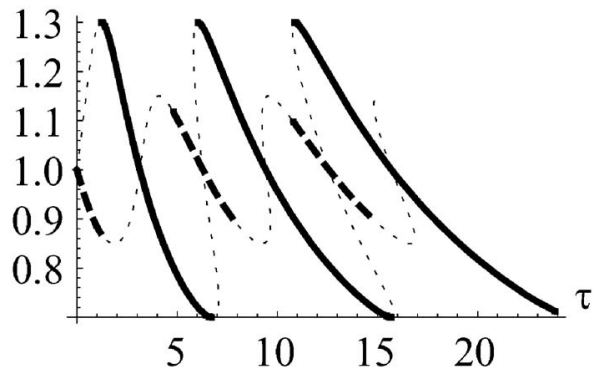

FIG. 12. The locking frequencies, as given by Eq. (24), are shown as a function of the delay $\tau$, for three bidirectionally coupled Kuramoto oscillators. The full curve corresponds to stable in-phase solutions, the dashed curve to stable out-of-phase solutions. Stability was calculated using Eqs. (3) and (26). The coupling strength has been chosen as $\kappa=-0.3$.

set of parameters than the out-of-phase state, and there are large regions where only in-phase synchronization is possible, even for moderate coupling and delay.

A ring of four bidirectionally coupled Kuramoto oscillators can only show two different states, the symmetric $(\Delta \phi$ $=0)$ and an antiphase state $(\Delta \phi=\pi)$. In the latter case oscillators one and three are in phase with each other, and in antiphase with the two other oscillators. The stable frequencies for these states are exactly the same as for only two coupled elements. The same two states, with the same stable locking frequencies, also exist if the coupling is unidirectional. In contrast to the bidirectional ring of four elements, the unidirectional ring exhibits in addition two other antiphase states.

\section{DISCUSSION}

Networks of coupled semiconductor lasers are of particular interest in the context of coupled nonlinear oscillators, as they provide a well-controlled experimental test-bed, and promise novel applications in photonics. The coupling delay in those networks originates from the propagation time of light or electronic signals between them, which can become even larger than the internal intensity oscillations time scale of a semiconductor laser. So far, only very small networks

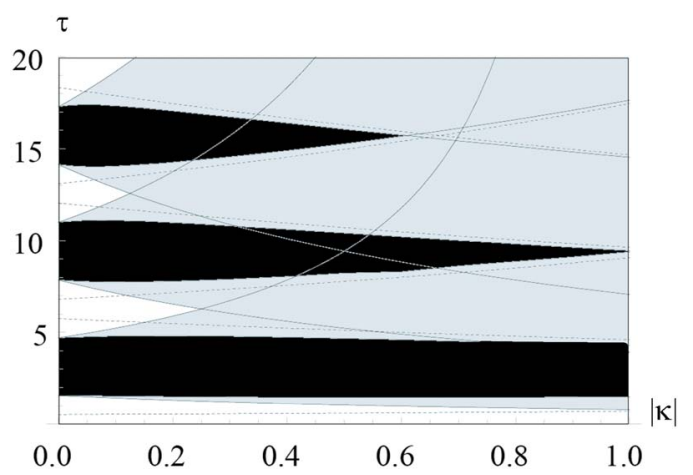

FIG. 13. (Color online) Stable regions in parameter space, for a ring of three bidirectionally coupled Kuramoto oscillators. Stability is calculated using Eqs. (24), (3), and (26). The dotted line shows the approximation of the Hopf bifurcation point Eq. (34) The coupling is repulsive. Black: only stable in-phase solutions exist; white: only stable antiphase solutions exist; gray: both types of solutions coexist. have been studied experimentally. In particular, configurations with two or three lasers have been investigated and discussed for applications in communication using chaotic carriers and key exchange. When two semiconductor lasers are coupled optically, the delay destabilizes the laser's output; the emission often even becomes chaotic. For small detuning and when the distance between the lasers is short, the system exhibits multistable locking, while for large detuning the emission shows self-sustained oscillations. ${ }^{35}$ When the lasers are coupled on long distance, the coupling induces complex dynamics. ${ }^{36,37}$ In addition, the network shows generalized synchronization: one laser is following the other with a time lag equal to the propagation time. Even if the lasers are operated under identical conditions (i.e., without detuning), symmetry is spontaneously broken. For two mutually coupled Kuramoto oscillators the symmetry-broken state (the out-of-phase state) is as probable as the state possessing the full symmetry (the in-phase state). The results on Kuramoto oscillators do not explain why the fully symmetric behavior does not occur in laser networks; but they indicate why the symmetry broken state is observed. In the laser network a time lag is measured; in the Kuramoto network the different states are characterized by a phase shift between the oscillators. While for periodic signals phase shift and time lag cannot be distinguished, the difference becomes essential when moving to chaotic dynamics as it is found for semiconductor lasers. The Kuramoto equations can be obtained as phase reduction of the equations modelling optically coupled semiconductor lasers; if we assume stable emission (i.e., constant and equal intensity and inversion for the entire laser network), we obtain Kuramoto equations describing the evolution of the phases of the electric fields of the semiconductor lasers. The natural frequency $\omega_{0}$ is then the optical frequency of the laser light and the coupling strength is related to the amount of light injected in the laser cavities and to the linewidth enhancement factor. The question then arises, as to how far the time lags in the case of full laser dynamics are linked to symmetry broken phase-shifted states in the Kuramoto model.

If one element is added to the chain, ${ }^{38}$ there is, surprisingly, no time lag in the synchronization between the outer lasers, despite the long propagation times. Even if the middle laser is detuned with respect to the outer ones, the outer elements remain in complete synchrony with each other. We have found the same synchronization pattern for the chain of Kuramoto oscillators. Based on these findings, it has been explored what the restrictions for the middle relay element are. It has been found that in order to obtain stable isochronal synchronization, it is sufficient to couple them through a semitransparent mirror, ${ }^{39}$ or, equivalently, to subject the two coupled lasers additionally to suitable delayed feedback. ${ }^{40}$ Isochronal synchronization has also been observed when the semiconductor lasers were coupled optoelectronically and subject to optoelectronical feedback. ${ }^{41}$ In analogy, in the Kuramoto network of two delay-coupled oscillators with feedback the most symmetric solution (the in-phase) is the most likely one. However, while in the laser network (for optical as well as for optoelectronical coupling) the feedback and coupling delay must match closely in order to obtain 
stable isochronal synchronization, this point is not critical for the Kuramoto oscillators.

A numerical study of a unidirectional ring and a bidirectionally coupled ring of three delay-coupled semiconductor lasers $^{42}$ supports the hypothesis that there is a link between time-shifts occurring in the dynamics of laser networks and phase shifts between Kuramoto oscillators. While in the bidirectional ring the output of the lasers is in complete synchronization, the unidirectional laser ring shows leaderlaggard behavior. This is also what the results on Kuramoto oscillators indicate.

Not only laser networks show similarities with the Kuramoto dynamics. In bidirectional rings of an even number of oscillators, two states are enhanced by the delay: the fully synchronized state and the state where each oscillator is in antiphase with its two neighbors. This second kind of network behavior, where an oscillator synchronizes with its second nearest neighbors, has also been found in networks of chaotic Bernoulli maps coupled with delay, ${ }^{43}$ where it is called "sublattice synchronization."

Another field where synchrony may have profound implications is neuroscience. Neuronal assemblies in distant regions in the brain have been experimentally observed to discharge synchronously, a dynamical state which has been hypothesized to be of relevance for information processing by the nervous system. ${ }^{19,44}$ It is a remarkable fact that such zero-time lag synchrony is maintained in spite of the axonal conduction delays accumulating along connections between two different brain areas. Our results support the view that given the proper topology some network motifs of oscillators can sustain zero-lag synchrony even in the presence of substantial coupling delays. Interestingly, some cerebral anatomical structures as the cortico-thalamo-cortical circuit resembles the motif composed by the three elements interacting bidirectionally in an open chain. ${ }^{45}$ This module has been analyzed in a previous section and observed to naturally enhance the zero-lag synchrony of their outer elements. The approach of decomposing complex brain networks into motifs in order to better understand its structural properties have been followed in Refs. 22 and 23. Here, we suggest that the investigation of synchrony patterns observed in complex neuronal circuits may also benefit from the study of synchronized solutions in network motifs.

\section{CONCLUSION}

We have studied small networks of Kuramoto oscillators with different topologies: open chains, with and without delayed feedback, as well as unidirectional and bidirectional rings. For the case of open chains we have found that two locked states are possible: synchronous states, where all the elements oscillate in phase with each other, and out-of-phase states, where the odd-numbered oscillators and the evennumbered oscillators differ by half a period. If the incoming signals are considered to scale with the degree of each node, which is often the case in real networks, the outer oscillators exhibit a small phase difference with respect to the inner ones due to boundary conditions. We have paid further attention to a chain of two elements, adding feedback. Here, the parameter ranges allowing stable out-of-phase states shrink considerably, while the synchronous state is stable over the same parameter range as in the case without feedback. Moreover, the out-of-phase locked state can lose stability in a Hopf bifurcation; a new state emerges, characterized by a phase difference between the nodes oscillating around $\pi$.

Without coupling delay, the unidirectional and bidirectional ring of Kuramoto oscillators show the same states with the same stability (but with different locking frequencies). The influence of delay is then shown to be different for both network symmetries. While in a unidirectional ring a coupling delay mainly acts as an inducer of multistability, it additionally enhances the more symmetric behavior in bidirectional rings.

In this study we have concentrated on the most symmetric situations of identical individual elements. The influence of parameter mismatch and noise, being essential for the understanding of real systems, will be addressed in the near future. In some other networks, a small mismatch of parameters of the nodes was observed not to destroy synchronous behavior completely. However, the resulting synchronization may be weaker, as the nodes do not show exactly the same dynamics. In an open chain of three lasers, a mismatch of the internal laser parameters of the outer lasers results in a lower cross correlation. ${ }^{38}$ When two lasers are mutually coupled through a mirror, stable isochronal synchronization is possible over a large range of coupling and feedback strengths, ${ }^{40}$ but when coupling delay and feedback delay do not closely match, only achronal synchronization is achieved. In networks of coupled logistic maps a parameter mismatch of the nodes causes a dispersion around the synchronized state. ${ }^{16}$ Our first numerical simulations with slightly detuned Kuramoto oscillator networks indicate that the elements still show periodic (frequency-locked) solutions, but the synchronization is also weaker, as the phase differences differ slightly from those of the symmetric network. Like in logistic map networks, ${ }^{16}$ this difference tends to diminish with growing coupling strength.

Although the simple Kuramoto oscillator networks cannot capture the full complexity of the dynamics of many real delay-coupled elements, some symmetry properties related to the interconnection pattern, are already covered. Agreements with the synchronization patterns occurring in delay-coupled semiconductor lasers can be found; in networks where symmetric (in-phase) and symmetry-broken (antiphase) states are equally probable, like for two mutually coupled elements and unidirectional rings, a symmetry breaking is observed in the dynamics of the laser system. In addition, if the most symmetric system is enhanced by the delay in Kuramoto oscillator networks, as we have found for the feedback system, for odd-numbered bidirectional rings and between the outer elements of a chain of three, isochronal synchronization may be observed in the corresponding laser network.

The comparison of delay-coupled Kuramoto oscillators with neuronal and semiconductor laser networks is a first step towards a hierarchy of models for delay-coupled networks of dynamical elements; this hierarchy allows us to explore which delay-induced properties originate from the network symmetry, which from phase or amplitude dynamics and which effects find their origin in a particular dynamical 
feature of each individual node. Some of these questions are left for future investigation.

\section{ACKNOWLEDGMENTS}

Part of this work has been performed at the Instituto de Fisica Interdisciplinar y Sistemas Complejos (IFISC), Universtitat de les Illes Balears, Palma de Mallorca, Spain, and we wish to thank Claudio Mirasso for his hospitality and guidance. We thank Paul Woafo, University of Yaounde I, Cameroon, for carefully reading the manuscript and for helpful suggestions. O.D. acknowledges the Research Foundation Flanders (FWO-Vlaanderen) for her fellowship and for project support. R.V. acknowledges the support of the Hertie Foundation. T.E. acknowledges the support of the Fonds National de la Recherche Scientifique (FNRS, Belgium). This work was partially supported by the Belgian Science Policy Office under Grant No. IAP-VI10 "photonics@be" and by the European Community Project GABA (FP6-NEST Contract No. 043309).

\section{APPENDIX: APPROXIMATION OF THE HOPF BIFURCATION POINT FOR THE ANTIPHASE STATES IN A BIDIRECTIONAL RING}

The stability of an antiphase state (characterized by the phase difference $\Delta \phi$ between neighboring oscillators) is determined by the characteristic equation (26),

$$
\begin{aligned}
\lambda= & \kappa \cos \Delta \phi \cos \omega \tau\left[-1+e^{-\lambda \tau}\left(\cos \frac{2 k \pi}{n}\right.\right. \\
& \left.\left.+i \tan \omega \tau \tan \Delta \phi \sin \frac{2 k \pi}{n}\right)\right],
\end{aligned}
$$

with $0<k<n$.

\section{The limit of small coupling strength}

We first explore the limit of small coupling strength $\kappa$. Assuming

$$
\begin{aligned}
& \lambda=\kappa \Lambda=\kappa\left[\lambda_{1}+\kappa \lambda_{2}+O\left(\kappa^{2}\right)\right], \\
& \tau_{\text {Hopf }}=\tau_{0}+\kappa \tau_{1}+O\left(\kappa^{2}\right), \\
& \omega=1-\kappa \cos \Delta \phi \sin \tau_{0}+O\left(\kappa^{2}\right),
\end{aligned}
$$

we obtain in lowest order in $\kappa$,

$$
\begin{aligned}
\lambda_{1}= & \cos \Delta \phi \cos \tau\left(\cos \frac{2 k \pi}{n}-1\right) \\
& +i \sin \tau \sin \Delta \phi \sin \frac{2 k \pi}{n} .
\end{aligned}
$$

A Hopf bifurcation occurs if $\tau_{0}=\pi / 2+m \pi$. To find the effect of the coupling strength $\kappa$ to the Hopf bifurcation delay $\tau_{\text {Hopf }}$, we determine the next order correction. Equation (A1) becomes

$$
\begin{aligned}
\lambda_{2}= & \cos \Delta \phi\left(1-\cos \frac{2 k \pi}{n}\right)\left(\tau_{1}-\tau_{0} \cos \Delta \phi\right) \\
& -i \lambda_{1} \tau_{0} \sin \Delta \phi \sin \frac{2 k \pi}{n} \\
= & \cos \Delta \phi\left(1-\cos \frac{2 k \pi}{n}\right)\left(\tau_{1}-\tau_{0} \cos \Delta \phi\right) \\
& +\tau_{0} \sin ^{2} \Delta \phi \sin ^{2} \frac{2 k \pi}{n} .
\end{aligned}
$$

A Hopf bifurcation occurs if

$$
\tau_{1}=\tau_{0}\left[1+\kappa \sin \tau_{0} \frac{\cos ^{2} \Delta \phi-\left(1+\cos \frac{2 k \pi}{n}\right) \sin ^{2} \Delta \phi}{\cos \Delta \phi}\right] .
$$

The factor $\kappa \sin \tau_{0} / \cos \Delta \phi$ is always positive for an upper boundary (in terms of $\tau$ ) and negative for a lower boundary for the delay time. The Hopf bifurcation in the direction $[1, \exp (2 \pi i / n), \ldots]$ will mark the stability boundary, as for $k=1$ the factor $\cos ^{2} \Delta \phi-(1+\cos 2 k \pi / n) \sin ^{2} \Delta \phi$ is smallest. The stability boundary is thus given by

$$
\tau_{\text {Hорf }}=\left(\frac{\pi}{2}+m \pi\right)\left[1 \pm \kappa \frac{\cos ^{2} \Delta \phi-\left(1+\cos \frac{2 \pi}{n}\right) \sin ^{2} \Delta \phi}{\cos \Delta \phi}\right] .
$$

\section{The limit of long delays}

Next, we explore the limit of long delays and large coupling. Assuming $\lambda=\kappa \Lambda$, we obtain

$$
\begin{aligned}
\Lambda= & \cos \Delta \phi \cos \omega \tau\left[-1+e^{-\kappa \Lambda \tau}\left(\cos \frac{2 k \pi}{n}\right.\right. \\
& \left.\left.+i \tan \omega \tau \tan \Delta \phi \sin \frac{2 k \pi}{n}\right)\right] .
\end{aligned}
$$

We insert in Eq. (A6) a pure imaginary eigenvalue $\Lambda=i \sigma$,

$$
\begin{aligned}
i \sigma= & \cos \Delta \phi \cos \omega \tau\left[1-e^{-i \kappa \sigma \tau}\left(\cos \frac{2 k \pi}{n}\right.\right. \\
& \left.\left.+i \tan \omega \tau \tan \Delta \phi \sin \frac{2 k \pi}{n}\right)\right] .
\end{aligned}
$$

If we assume $\kappa \sigma \tau$ is of order 1 , we can neglect $\sigma$. This leads to

$$
e^{i \sigma \tau}=\cos \frac{2 k \pi}{n}+i \sin \frac{2 k \pi}{n} \tan \omega \tau \tan \Delta \phi,
$$

which is solved for $|\tan \omega \tau \tan \Delta \phi|=1$ and $\kappa \sigma \tau= \pm 2 k \pi / n$.

\footnotetext{
${ }^{1}$ D. Watts and S. Strogatz, Nature (London) 393, 440 (1998).

${ }^{2}$ A. Barabasi and R. Albert, Science 286, 509 (1999).

${ }^{3}$ S. Strogatz, Nature (London) 410, 268 (2001).

${ }^{4}$ R. Albert and A. Barabasi, Rev. Mod. Phys. 74, 47 (2002).

${ }^{5}$ F. Atay, J. Jost, and A. Wende, Phys. Rev. Lett. 92, 144101 (2004).

${ }^{6}$ A. Martí, M. Ponce, and C. Masoller, Physica A 371, 104 (2006).
} 
${ }^{7}$ A. Pikovky, M. Rosenblum, and J. Kurths, Synchronization: A Universal Concept in Nonlinear Sciences (Cambridge University Press, Cambridge, 2003).

${ }^{8}$ L. M. Pecora, T. L. Carroll, G. Johnson, D. Mar, and J. Heagy, Chaos 7, 520 (1997)

${ }^{9}$ W. Singer, Nature (London) 397, 391 (1999).

${ }^{10}$ F. Varela, J. Lachaux, E. Rodriguez, and J. Martinerie, Nat. Rev. Neurosci. 2, 229 (2001).

${ }^{11}$ D. Welch, B. Chan, and W. Streifer, Electron. Lett. 24, 113 (1988).

${ }^{12}$ H. Winful and L. Rahman, Phys. Rev. Lett. 65, 1575 (1990).

${ }^{13}$ K. Wiesenfeld, P. Colet, and S. Strogatz, Phys. Rev. Lett. 76, 404 (1996).

${ }^{14}$ G. Filatrella, N. Pedersen, and K. Wiesenfeld, Phys. Rev. E 61, 2513 (2000).

${ }^{15}$ C. Masoller, A. Marti, and D. Zanette, Physica A 325, 186191 (2003).

${ }^{16}$ A. Marti and C. Masoller, Physica A 342, 344350 (2004).

${ }^{17}$ C. Masoller and A. Martí, Phys. Rev. Lett. 94, 134102 (2005).

${ }^{18}$ Z. Neda, E. Ravasz, Y. Brechet, T. Vicsek, and A. Barabasi, Nature (London) 403, 849 (2000).

${ }^{19}$ P. R. Roelfsma, A. Engel, P. König, and W. Singer, Nature (London) 385, 157 (1997).

${ }^{20}$ A. Argyris, D. Syvridis, L. Larger, V. Annovazzi-Lodi, P. Colet, I. Fischer, J. Garcia-Ojalvo, C. Mirasso, and L. Pesquera, Nature (London) 437, 343 (2005).

${ }^{21}$ R. Milo, S. Shen-Orr, S. Itzkovitz, N. Kashtan, D. Chklovskii, and U. Alon, Science 298, 824 (2002).

${ }^{22}$ O. Sporns and R. Kötter, PLoS Biol. 2, e369 (2004).

${ }^{23}$ R. Prill, P. Iglesias, and A. Levchenko, PLoS Biol. 3, e343 (2005).

${ }^{24}$ H. G. Schuster and P. Wagner, Prog. Theor. Phys. 81, 939 (1989).

${ }^{25}$ D. V. Ramana Reddy, A. Sen, and G. L. Johnston, Phys. Rev. Lett. 85, 3381 (2000).

${ }^{26}$ M. Golubitsky and I. Stewart, The Symmetry Perspective (Birkhauser, Boston, 2003).
${ }^{27}$ J. Wu, Trans. Am. Math. Soc. 350, 4799 (1998).

${ }^{28}$ F. Atay and T. Biyitoglu, Phys. Rev. E 72, 016217 (2005).

${ }^{29}$ G. Osipov, J. Kurths, and C. Zhou, Synchronization in Oscillatory Networks (Springer, Berlin, 2007).

${ }^{30}$ Y. Kuramoto, Int. J. Bifurcation Chaos Appl. Sci. Eng. 7, 789 (1997).

${ }^{31}$ H. Daido, Int. J. Bifurcation Chaos Appl. Sci. Eng. 7, 807 (1997).

${ }^{32}$ M. Yeung and S. Strogatz, Phys. Rev. Lett. 82, 648 (1999).

${ }^{33}$ G. Kozyreff, A. Vladimirov, and P. Mandel, Phys. Rev. Lett. 85, 3809 (2000).

${ }^{34}$ M. Earl and S. Strogatz, Phys. Rev. E 67, 036204 (2003).

${ }^{35}$ H.-J. Wünsche, S. Bauer, J. Kreissl, O. Ushakov, N. Korneyev, F. Henneberger, E. Wille, H. Erzgraber, M. Peil, W. Elsäßer, and I. Fischer, Phys. Rev. Lett. 94, 163901 (2005).

${ }^{36}$ T. Heil, I. Fischer, W. Elsasser, J. Mulet, and C. Mirasso, Phys. Rev. Lett. 86, 795 (2001).

${ }^{37}$ J. Mulet, C. Mirasso, T. Heil, and I. Fischer, J. Opt. B: Quantum Semiclassical Opt. 6, 97 (2004).

${ }^{38}$ I. Fischer, R. Vicente, J. Buldu, M. Peil, C. Mirasso, M. Torrent, and J. Garcia-Ojalvo, Phys. Rev. Lett. 97, 123902 (2006).

${ }^{39}$ R. Vicente, C. Mirasso, and I. Fischer, Opt. Lett. 32, 403 (2007).

${ }^{40}$ E. Klein, N. Gross, M. Rosenbluh, W. Kinzel, L. Khaykovich, and I. Kanter, Phys. Rev. E 73, 066214 (2006).

${ }^{41}$ R. Vicente, S. Tang, J. Mulet, C. Mirasso, and J.-M. Liu, Phys. Rev. E 73, 047201 (2006).

${ }^{42}$ J. Buldu, M. Torrent, and J. Garcia-Ojalvo, J. Lightwave Technol. 25, 1549 (2007)

${ }^{43}$ J. Kestler, W. Kinzel, and I. Kanter, Phys. Rev. E 76, 035202 (2007).

${ }^{44}$ M. Castelo-Branco, R. Goebel, S. Neuenschwander, and W. Singer, Nature (London) 405, 685 (2000).

${ }^{45}$ E. Jones, Philos. Trans. R. Soc. London, Ser. B 357, 1659 (2002). 